\title{
An Organocatalyzed and Ultrasound Accelerated Expeditious Synthetic Route to 1,5-Benzodiazepines under Solvent-Free Conditions
}

\author{
Pravin V. Shinde, Bapurao B. Shingate, and Murlidhar S. Shingare* \\ Department of Chemistry, Dr. Babasaheb Ambedkar Marathwada University, Aurngabad-431 004, India \\ *E-mail: prof msshingare@rediffmail.com \\ Received January 15, 2010, Accepted February 1, 2011
}

\begin{abstract}
In the present work, successful implementation of ultrasound irradiations for the rapid synthesis of 1,5benzodiazepine derivatives under solvent-free conditions is demonstrated. Use of a novel catalyst i.e. camphor sulphonic acid in combination with ultrasound technique is reported for the first time. Comparative study for the synthesis of 1,5-benzodiazepines using conventional as well as ultrasonication method is discussed.
\end{abstract}

Key Words : 1,5-Benzodiazepine, Camphor sulphonic acid, Ultrasonication, Solvent-free reaction.

\section{Introduction}

Emerging area of green chemistry demands ecofriendly organic chemical processes, considering the increasing environmental pollution and its drastic impact on living systems. ${ }^{1}$ Hence, significance of greener pathways in organic synthesis is ever-growing in order to attain the sustainability. For many chemical operations, a major adverse effect to the environment is consumption of energy for heating and cooling. To overcome such problems, it is highly enviable to develop efficient routes that utilize alternative energy resources. ${ }^{2}$ In this regard, number of organic chemists are engaged in the development of cleaner and ecofriendly synthetic protocols. One of the thrust areas for achieving rapid syntheses of organic compounds minimizing the need of conventional heating and cooling, and use of hazardous/ volatile organic solvents involves the utilization of nonclassical energy resources like ultrasound irradiation technique and microwave energy. ${ }^{3}$

Ultrasound technique has increasingly been used in organic synthesis and therefore acting as a stepping stone towards green chemistry. Simple experimental procedure, very high yields, increased selectivities and clean reaction of many ultrasound induced organic transformations offer additional convenience in the field of synthetic organic chemistry. One of the most promising features of this technique is that the assistance of ultrasonic irradiation efficiently shortens the reaction times and the driving force behind this is the phenomenon of acoustic cavitation. ${ }^{4}$ Ultrasound irradiations also keep the potential to facilitate organic transformations at ambient conditions by eliminating the need of drastic conditions such as temperature, pressure and concentrations. ${ }^{5}$ Furthermore, considering the basic green chemistry concepts, ultrasound is proving to be more efficient for improving the traditional reactions that require expensive reagents, strong acidic conditions, longer reaction time, high temperatures, unsatisfactory yields and incompatibility with other functional groups. ${ }^{6,7}$ Therefore, there is a lot of scope for the use of ultrasound technique in the organic synthesis and in this endeavor organic chemists are focusing more and more on its use for the synthesis of bioactive molecules.

Benzodiazepines constitute an important class of biodynamic heterocycles and synthesis of these compounds has been receiving great attention in the field of medicinal and pharmaceutical chemistry owing to their broad spectrum of biological/pharmacological activities ${ }^{8}$ and their often use as analgesic, sedative, hypnotic, anti-consultant, anti-anxiety, anti-depressive, and anti-inflammatory agents. ${ }^{9}$ In addition, 1,5-benzodiazepines are valuable synthetic intermediates for the preparation of other heterocyclic compounds such as triazolo-, oxadiazolo-, oxazino-, furano-, and quinazolinobenzodiazepines. ${ }^{10}$

Cyclocondensation of $o$-phenylenediamines with carbonyl compounds is one of the well established synthetic methods for the construction of 1,5-benzodiazepine derivatives. ${ }^{11} \mathrm{~A}$ wide range of catalysts, such as $\mathrm{BF}_{3} \cdot \mathrm{OEt}_{2},{ }^{12}$ polyphosphoric acid, ${ }^{13} \mathrm{CeCl}_{3}-\mathrm{NaI} / \mathrm{SiO}_{2},{ }^{14} \mathrm{I}_{2},{ }^{15} \mathrm{ZnCl}_{2},{ }^{16} \mathrm{SmI}_{2},{ }^{17} \mathrm{YbCl}_{3},{ }^{18}$ $\mathrm{MgO} / \mathrm{POCl}_{3},{ }^{19}$ Amberlyst-15, ${ }^{20} \mathrm{Yb}(\mathrm{OTf})_{3},{ }^{21} \mathrm{Ga}(\mathrm{OTf})_{3},{ }^{22}$ $\mathrm{Al}_{2} \mathrm{O}_{3} / \mathrm{P}_{2} \mathrm{O}_{5},{ }^{23} \mathrm{AcOH} / \mathrm{MW},{ }^{24}$ sulfated zirconia, ${ }^{25} \mathrm{NBS},{ }^{26}$ cerium ammonium nitrate (CAN) ${ }^{27}$ montmorillonite $\mathrm{K} 10,{ }^{28}$ $\mathrm{Ag}_{3} \mathrm{PW}_{12} \mathrm{O}_{40},{ }^{29} \mathrm{InBr}_{3} / \mathrm{InCl}_{3},{ }^{30}$ and ionic liquids, ${ }^{31}$ have been utilized for this transformation, alongwith their own merits and demerits. In this regard, few eco-friendly routes have also been reported in literature. ${ }^{32}$ Since, 1,5-benzodiazepine derivatives keeps enormous significance in pharmaceutical and medicinal fields, the development of simple, eco-benign and low cost protocol is still desirable.

Interest in the field of organocatalysis has increased spectacularly in the last few years as a result of both the novelty of the concept and, more importantly, the fact that the efficiency and selectivity of many organocatalytic reactions meet the standards of established organic reactions. ${ }^{33}$ Camphor sulphonic acid (CSA) is achieving enormous significance in organic synthesis as this catalyst is used in the synthesis of chromans, ${ }^{34 \mathrm{a}}$ ligands, ${ }^{34 \mathrm{~b}}$ and pseudoglycosides, ${ }^{34 \mathrm{c}}$ as an auxillary, ${ }^{35 \mathrm{a}}$ and in some polymerization reactions. ${ }^{35 b}$ Considering the significance of all above discussed aspects and in continuation of our endeavor ${ }^{36}$ towards the 
development of ecofriendly synthetic protocols for the construction of bioactive molecules, it was thought worthwhile to develop a new, simple, greener and expeditious route for the synthesis of 1,5-benzodiazepine derivatives under conventional/ultrasound technique using camphor sulphonic acid as an organocatalyst.

\section{Results and Discussion}

For the reasons of economy and pollution, solvent-free methods are of great interest in order to modernize classical procedures making them more clean, safe and easy to perform. ${ }^{37}$ Therefore, emphasis was given to carry out all the optimization experiments under solvent-free conditions at room temperature.

In our initial experiments towards the development of efficient methodology for the synthesis of 1,5-benzodiazepines, reaction of $o$-phenylenediamine with acetophenone was performed under solvent-free and catalyst-free conditions (Scheme 1). But, unfortunately even after $60 \mathrm{~min}$ reaction was not initiated and starting materials were recovered. Since, uncatalyzed reaction did not lead to the formation of product, it was thought that for initiation of the reaction intervention of catalyst is must. Hence, various well known acid catalysts such as boric acid, sulfamic acid, sulfanilic acid, EDTA-2Na salt in water and $p$ TSA as well as some organocatalyts i.e. L-proline and camphor sulphonic acid were tested to activate the reaction mass.

When sulphamic and sulfanilic acid was used for the reaction, desired product was obtained in only $59 \%$ and $66 \%$ yields respectively (Table 1, entries 2 and 3). EDTA.2Na failed to give the product in desirable yield (Table 1, entry 5). In contrast, Boric acid and oxalic acid delivered the product in good yields (Table 1, entries 1 and 4). Although boric and oxalic acid were found to be effective catalysts for the model reaction, some organocatalysts like L-proline and camphor sulphonic acid were also examined in order to improve efficiency of the results obtained in the initial study. To our surprise, reaction in the presence of CSA found to proceed rapidly affording excellent $89 \%$ yield (Table 1, entry 11). L-proline also delivered the product in good yield (Table 1, entry 7), but in comparison to CSA it was in less extent. Therefore, considering the effective catalytic activity of CSA and for exploitation of its applications in organic transformations, CSA was preferred as a catalyst of choice for subsequent optimization studies.

To determine the exact requirement of catalyst for the reaction, we investigated the model reaction using different concentrations of CSA such as 1, 2.5, 5, 10 and $15 \mathrm{~mol} \%$. During this study, formation of the product was observed in

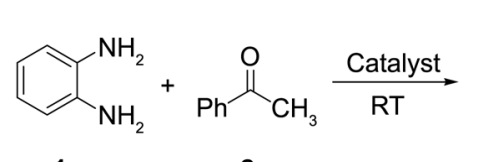

$1 \mathrm{a}$

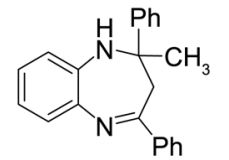

3a
Scheme 1. Standard model reaction.
$39,57,77,89$ and $90 \%$ yield respectively (Table 1 , entries 8 12). This indicated that $10 \mathrm{~mol} \%$ of CSA was sufficient to carry out the reaction smoothly.

It is worthy to point out here, since CSA is having carbonyl group in its structure one may speculate the possibility of the reaction of CSA with $o$-phenylenediamine. To confirm this, a series of experiments were performed using CSA in 5, 10,20 and $50 \mathrm{~mol} \mathrm{\%}$ in the absence of second reactant viz. ketone. But, even after $60 \mathrm{~min}$ initiation of the reaction was not observed and starting materials were recovered as it is. In this way, possibility of the side reaction of CSA and $o$-phenylenediamine has been ruled out via above study.

Non-conventional energy resources like ultrasound and microwave irradiation techniques have become very popular and useful chemical technologies in organic chemistry. Considering the well established applications of ultrasound and microwave activation to promote variety of chemical reactions, we next attempted to carry out the model reaction using optimized reaction conditions under ultrasound as well as microwave irradiations with a view to explore whether, (i) the reaction could be expedited and, (ii) the product yield could be enhanced. Assistance of ultrasound irradiations did not brought significant improvement in the product yield (91\%), Table 1 , entry 13 but it is noteworthy to point out that the reaction time reduced enormously to $15 \mathrm{~min}$ as compared to conventional method (50 min). In contrast, reaction under microwave irradiations failed to afford the product in good yields (Table 1, entry 14). This practically observed fall in the yield may be due to the hydrolysis of benzodiazepine in a microwave oven. ${ }^{38}$

It should be noted that, above study clarifies the assistance of ultrasound energy to accelerate the rate of reaction. The difference in yields and reaction times may be a consequence of the specific effects of ultrasound. The effect observed on

Table 1. Screening of catalysts ${ }^{a}$

\begin{tabular}{|c|c|c|c|c|}
\hline Entry & Solid promoter & $\begin{array}{c}\text { Catalyst } \\
\text { concentration } \\
(\mathrm{mol} \%)\end{array}$ & $\begin{array}{l}\text { Time } \\
(\min )\end{array}$ & $\begin{array}{c}\text { Yield }^{b} \\
(\%)\end{array}$ \\
\hline 1 & Boric acid & 10 & 60 & 79 \\
\hline 2 & Sulphamic acid & 10 & 120 & 59 \\
\hline 3 & Sulfanilic acid & 10 & 120 & 66 \\
\hline 4 & Oxalic acid & 10 & 120 & 81 \\
\hline 5 & EDTA·2Na (In water $(1 \mathrm{~mL}))$ & 10 & 120 & 35 \\
\hline 6 & $p$ TSA & 10 & 60 & 74 \\
\hline 7 & L-proline & 10 & 60 & 78 \\
\hline 8 & CSA & 1 & 60 & 39 \\
\hline 9 & CSA & 2.5 & 60 & 57 \\
\hline 10 & CSA & 5 & 60 & 77 \\
\hline 11 & CSA & 10 & 60 & 89 \\
\hline 12 & CSA & 15 & 60 & 90 \\
\hline 13 & $\mathrm{CSA}^{c}$ & 10 & 15 & 91 \\
\hline 14 & $\mathrm{CSA}^{d}$ & 10 & 15 & 65 \\
\hline
\end{tabular}

${ }^{\mathrm{a}}$ Reaction conditions: $\mathbf{1 a}(1 \mathrm{mmol})$ and $\mathbf{2 a}(2 \mathrm{mmol})$ at RT under solvent free conditions. ${ }^{b}$ Isolated yields. ${ }^{c}$ Under ultrasound irradiation. ${ }^{d}$ Under microwave irradiation. 

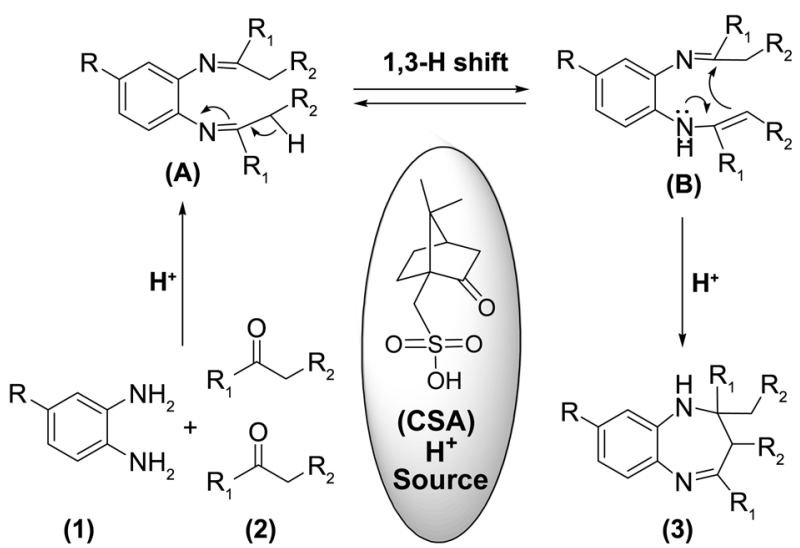

(B)

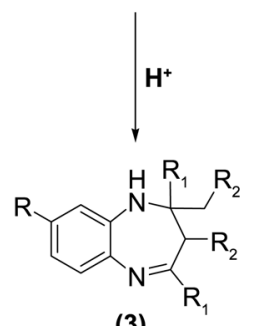

(3)

Figure 1. A plausible mechanism involved in the synthesis of 1,5benzodiazepine.

the reaction is due to the phenomenon of acoustic cavitation, a physical process that creates, enlarges, and implodes gaseous and vaporous cavities in an irradiated liquid, thus enhancing the mass transfer and allowing chemical reactions to occur swiftly. The creation of the so-called hot spots in the reaction mixture produces intense local temperatures and high pressures generated inside the cavitation bubble and its interfaces when it collapses. ${ }^{4-7}$ The collapse of cavitation bubbles result in the formation very reactive chemical species having short lifetime which facilitates the rapid synthesis of 1,5-benzodiazepines.

A possible mechanism involved in CSA catalyzed cyclocondensation reaction for the synthesis of 1,5-benzodiazepines can be outlined as follows: $o$-phenylenediamine (1) reacts with two molecules of ketone (2) in the presence of camphor suphonic acid as a catalyst to form schiff base (A), which undergoes 1,3-H shift and get converted into intermediate (B). Thus formed intermediate (B) via subsequent cyclization affords the final product (3), i.e., 1,5-benzodiazepine. Diagrammatic representation of the mechanism is rationalized in Figure 1.

Having established the optimum experimental conditions for obtaining the best yields of 1,5-benzodiazepine derivatives, different $o$-phenylenediamines with respect to various ketones were examined under conventional and ultrasound method. Notably, all the substrates were observed to be well tolerated under optimized conditions furnishing the product in good to excellent yields. All the results are compiled in Table 2 . Formation of the desired product was confirmed by comparing their physical constant, IR, ${ }^{1} \mathrm{H}$ NMR, ${ }^{13} \mathrm{C}$ NMR and mass spectro- scopic data with reported compounds.

\section{Conclusions}

In summary, an efficient, greener and expeditious synthetic protocol for 1,5-benzodiazepines has been developed. This synthetic strategy offers some remarkable advantages, such as use of non-classical energy source (ultrasonication), non-toxic and economically viable catalyst, solvent-free conditions, high isolated yields, and more importantly
Table 2. Synthesis of 1,5-Benzodiazepine Derivatives ${ }^{a}$

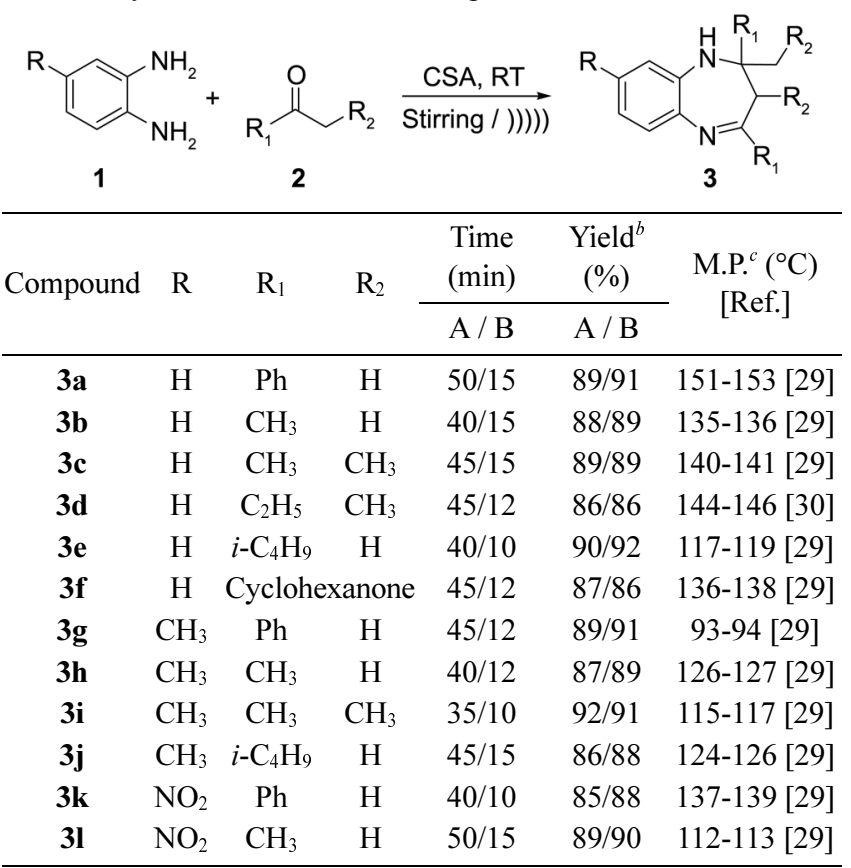

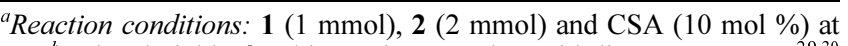
RT. ${ }^{b}$ Isolated yields. ${ }^{c}$ Melting points matches with literature reports. ${ }^{99,30}$ $\mathbf{A}=$ Conventional stirring method; $\mathbf{B}=$ Ultrasound irradiation method.

reduced reaction times. Present work is the first report on the combined use of ultrasound irradiation and solvent-free conditions for the synthesis of 1,5-benzodiazepines.

\section{Experimental}

General. All chemicals were purchased and used without any further purification. Melting points were determined on a Veego apparatus and are uncorrected. Infrared spectra were recorded on a Bruker spectrophotometer in a $\mathrm{KBr}$ disc, and the absorption bands are expressed in $\mathrm{cm}^{-1} .{ }^{1} \mathrm{H}$ NMR spectra were recorded on NMR spectrometer Varian AS $400 \mathrm{MHz}$ in DMSO- $d_{6}$, chemical shifts $(\delta)$ are in ppm relative to TMS. Mass spectra were taken on a Macro mass spectrometer (Waters) by electro-spray method (ES). Bandelin Sonorex (with a frequency of $35 \mathrm{KHz}$ and a nominal power $200 \mathrm{~W}$ ) ultrasonic bath was used for ultrasonic irradiation with Builtin heating, $30-80{ }^{\circ} \mathrm{C}$ thermostatically adjustable. The reaction vessel was placed inside the ultrasonic bath containing water. Microwave oven equipped with a turntable was used (LG Smart Chef MS-255R operating at $2450 \mathrm{MHz}$ having maximum output of $900 \mathrm{~W}$ ) for reaction.

Typical Experimental Procedure.

Conventional Method: A mixture of $o$-phenylenediamine 1a (110 mg, $1 \mathrm{mmol})$, acetophenone $\mathbf{2 a}(240 \mathrm{mg}, 2 \mathrm{mmol})$ and camphor sulphonic acid $(23 \mathrm{mg}, 10 \mathrm{~mol} \%$ ) in a closed round bottomed flask of capacity $25 \mathrm{~mL}$ was allowed to stir vigorously at room temperature. Reaction progress was monitored by TLC (ethyl acetate/n-hexane, 2:8). After completion of the reaction, reaction mixture was poured on icecold water and stirred well. Thus obtained yellow coloured 
solid product was collected by simple filtration, washed with water and dried. This crude product (3a) was then recrystallized from aqueous ethanol to get pure product.

Ultrasound Method: A mixture of $o$-phenylenediamine $1 \mathbf{a}(110 \mathrm{mg}, 1 \mathrm{mmol})$, acetophenone 2a (240 mg, $2 \mathrm{mmol})$ and camphor sulphonic acid (23 mg, $10 \mathrm{~mol} \%$ ) in a closed round bottomed flask of capacity $25 \mathrm{~mL}$ was subjected to ultrasound irradiation in ultrasonic bath at room temperature. Reaction progress was monitored by TLC (ethyl acetate $/ n$-hexane, $2: 8)$. After completion of the reaction, reaction mixture was poured on ice-cold water and stirred well. Thus obtained yellow coloured solid product was collected by simple filtration, washed with water and dried. This crude product (3a) was then recrystallized from aqueous ethanol to get pure product.

\section{Spectral Data for Representative Compounds.}

2-Methyl-2,4-diphenyl-2,3-dihydro-1H-1,5-benzodiazepine (3a): Yellow solid; ${ }^{1} \mathrm{H}$ NMR (DMSO- $\left.d_{6}, 400 \mathrm{MHz}\right) \delta$ $1.63(\mathrm{~s}, 3 \mathrm{H}), 2.85$ (d, 1H, $J=14 \mathrm{~Hz}), 3.27$ (d, 1H, J=14 Hz), 5.66 (brs, 1H, -NH), 6.81-6.86 (m, 1H, Ar-H), 7.01 (d, 2H, J $=7.6 \mathrm{~Hz}, \mathrm{Ar}-\mathrm{H}), 7.10$ (dd, 2H, $J=1.6$ and $2.8 \mathrm{~Hz}, \mathrm{Ar}-\mathrm{H})$, 7.18 (t, 2H, $J=8.0 \mathrm{~Hz}, \mathrm{Ar}-\mathrm{H}), 7.23-7.31$ (m, 3H, Ar-H), 7.52 (d, 2H, $J=7.2 \mathrm{~Hz}, \mathrm{Ar}-\mathrm{H}), 7.66$ (d, 2H, $J=7.6 \mathrm{~Hz}, \mathrm{Ar}-\mathrm{H})$; IR $\left(\mathrm{KBr}, \mathrm{cm}^{-1}\right) v 3352,1648,1597 ;{ }^{13} \mathrm{C}$ NMR (DMSO- $d_{6}, 100$ MHz) $\delta 29.5,43.1,72.9,121.5,121.8,125.6,126.1,126.7$, $127.0,127.6,128.5,128.8,129.4,137.5,139.3,139.9$, 147.0, 166.9; ES-MS: $313.2\left(\mathrm{M}^{+}\right)$.

2,4,4-Trimethyl-2,3-dihydro-1H-1,5-benzodiazepine (3b): Pale yellow solid; ${ }^{1} \mathrm{H}$ NMR (DMSO- $\left.d_{6}, 400 \mathrm{MHz}\right) \delta 1.22$ (s, $6 \mathrm{H}), 2.14$ (s, 2H), 2.20 (s, 3H), 4.69 (brs, 1H, -NH), 6.456.91 (m, 4H, Ar-H); IR (KBr, cm $\left.{ }^{-1}\right) v 3334,1645,1594 ;{ }^{13} \mathrm{C}$ NMR (DMSO- $\left.d_{6}, 100 \mathrm{MHz}\right) \delta 29.6,29.9,30.3,46.0,67.8$, 122.5, 122.9, 126.0, 127.7, 137.9, 140.6, 171.1; ES-MS: $189.1\left(\mathrm{M}^{+}\right)$.

10-Spirocyclohexan-2,3,4,10,11,11a-hexahydro-1 $H$-dibenzo $[\boldsymbol{b}, \boldsymbol{e}][\mathbf{1 , 4}]$ diazepine (3f): Pale yellow solid; ${ }^{1} \mathrm{H}$ NMR (DMSO- $\left.d_{6}, 400 \mathrm{MHz}\right) \delta 1.21-1.98(\mathrm{~m}, 16 \mathrm{H}), 2.42-2.66(\mathrm{~m}$, $3 \mathrm{H}), 4.69$ (brs, 1H, -NH), 6.33-3.37 (m, 2H, Ar-H), 6.456.49 (m, 2H, Ar-H); IR (KBr, cm $\left.{ }^{-1}\right) v 3308,1638,1601$; ESMS: $269.2\left(\mathrm{M}^{+}\right)$.

\section{References}

1. Anastas, P. T.; Kirchhoff, M. M. Acc. Chem. Res. 2002, 35, 686.

2. Sheldon, R. A. Green Chem. 2005, 7, 267.

3. (a) Nuchter, M.; Ondruschka, B.; Jungnickel, A.; Muller, U. $J$. Phys. Org. Chem. 2000, 13, 579. (b) Varma, R. S. Green Chem. 2008, 10,1129 and references cited therein.

4. Mason, T. J. Chem. Soc. Rev. 1997, 26, 443.

5. (a) Luche, J. L. Synthetic Organic Sonochemistry; Plenum Press: New York, 1998. (b) Mason, T. J. Advances in Sonochemistry; JAI Press: London and Greenwich, CT, 1990.

6. (a) Mason, T. J.; Lorimer, J. P. Chem. Soc. Rev. 1987, 16, 239. (b) Li, J. T.; Wang, S. X.; Chen, G. F.; Li, T. S. Curr. Org. Synth. 2005, 2, 415. (c) Cravotto, G.; Cintas, P. Chem. Soc. Rev. 2006, 35, 180. (d) Cella, R.; Stefani, H. Tetrahedron 2009, 65, 2619.

7. Mason, T. J.; Cintas, P. In Handbook of Green Chemistry and Technology; Clark, J., Macquarrie, D., Eds.; Blackwell Science: Oxford, 2002.

8. (a) Schutz, H. Benzodiazepines; Springer: Heidelberg, 1982. (b)
Landquist, J. K. In Comprehensive Heterocyclic Chemistry; Katritzky, A. R., Rees, C. W., Eds.; Pergamon: Oxford, 1984.

9. Randall, L. O.; Kappel, B. In Benzodiazepines; Garattini, S., Mussini, E., Randall, L. O., Eds.; Raven Press: New York, 1973.

10. (a) Essaber, M.; Baouid, A.; Hasnaoui, A.; Benharref, A.; Lavergne, J. P. Synth. Commun. 1998, 28, 4097. (b) Xu, J. X.; Wu, H. T.; Jin, S. Chin. J. Chem. 1999, 17, 84. (c) Zhang, X. Y.; Xu, J. X.; Jin, S. Chin. J. Chem. 1999, 17, 404. (d) Reddy, K. V. V.; Rao, P. S.; Ashok, D. Synth. Commun. 2000, 30, 1825. (e) Zhang, W.; Williams, J. P.; Lu, Y.; Nagashima, T.; Chu, Q. Tetrahedron Lett. 2007, 48, 563.

11. Ried, W.; Torinus, E. Chem. Ber. 1959, 92, 2902.

12. Herbert, J. A. L.; Suschitzky, H. J. Chem. Soc.: Perkin Trans. 1 1974, 2657.

13. Jung, D. I.; Choi, T. W.; Kim, Y. Y.; Kim, I. S.; Park, Y. M.; Lee, Y. G.; Jung, D. H. Synth. Commun. 1999, 29, 1941.

14. Sabitha, G.; Reddy, G. S.; Reddy, K. B.; Reddy, N. M.; Yadav, J. S. Adv. Synth. Catal. 2004, 346, 921.

15. (a) Bandgar, B. P.; Bettigeri, S. V.; Joshi, N. S. Synth. Commun. 2004, 34, 1447. (b) Chen, W. Y.; Lu, J. Synlett 2005, 8, 1337.

16. Pasha, M. A.; Jayashankara, V. P. Heterocycles 2006, 68, 1017.

17. Luo, Y. Q.; Xu, F.; Han, X. Y.; Shen, Q. Chin. J. Chem. 2005, 23 , 1417.

18. Wu, J. T.; Xu, F.; Zhou, Z. Q.; Shen, Q. Synth. Commun. 2006, 36, 457.

19. Balakrishna, M. S.; Kaboundin, B. Tetrahedron Lett. 2001, 42, 1127.

20. Yadav, J. S.; Reddy, B. V. S.; Eshwaraian, B.; Anuradha, K. Green Chem. 2002, 4, 592.

21. Curini, M.; Epifano, F.; Marcotullio, M. C.; Rosati, O. Tetrahedron Lett. 2001, 42, 3193.

22. (a) Pan, X. Q.; Zou, J. P.; Huang, Z. H.; Zhang, W. Tetrahedron Lett. 2008, 49, 5302. (b) Jiang, Y. J.; Cai, J. J.; Zou, J. P. Zhang, W. Tetrahedron Lett. 2010, 51, 471.

23. Kaboudin, B.; Navaee, K. Heterocycles 2001, 55, 1443.

24. Pozarentzi, M.; Stephanidou, S. J.; Tsoleridis, C. A. Tetrahedron Lett. 2002, 43, 1755.

25. Reddy, B. M.; Sreekanth, P. M. Tetrahedron Lett. 2003, 44, 4447.

26. Kuo, C. W.; More, S. V.; Yao, C. F. Tetrahedron Lett. 2006, 47, 8523.

27. Varala, R.; Enugala, R.; Nuvula, S.; Adapa, S. R. Synlett 2006, 7 , 1009.

28. An, L. T.; Ding, F. Q.; Zou, J. P.; Lu, X. H. Synth. Commun. 2008, $38,1259$.

29. Yadav, J. S.; Reddy, B. V. S.; Praveenkumar, S.; Nagaiah, K.; Lingaiah, N.; Saiprasad, P. S. Synthesis 2004, 6, 901.

30. Yadav, J. S.; Reddy, B. V. S.; Praveenkumar, S.; Nagaiah, K. Synthesis 2005, 3, 480.

31. Du, Y. Y.; Tian, F. L.; Zhao, W. Z. Synth. Commun. 2006, 36, 1661.

32. (a) Guzen, K. P.; Cella, R.; Stefani, H. A. Tetrahedron Lett. 2006, 47, 8133. (b) Sharma, S. D.; Gogoi, P.; Konwar, D. Green Chem. 2007, 9,153 .

33. Dalko, P. I.; Moisan, L. Angew. Chem. Int. Ed. 2004, 43, 5138.

34. (a) Makoto, M.; Yamamoto, H. Bull. Chem. Soc. Jpn. 1995, 68, 2657. (b) Gayet, A.; Bolea, C.; Andersson, P. G. Org. Biomol. Chem. 2004, 2, 1887. (c) Gorityala, B. K.; Cai, S.; Ma, J.; Liu, X. W. Bioorg. Med. Chem. Lett. 2009, 19, 3093.

35. (a) Barrett, A. G. M.; Braddock, D. C.; Christian, P. W. N.; Pilipauskas, D.; White, A. J. P.; Williams, D. J. J. Org. Chem. 1998, 63, 5818. (b) Sejin, O.; Gijung, K.; Narae, K.; Shim, S. E.; Soonja, C. Macromol. Res. 2005, 13, 187.

36. (a) Sapkal, S. B.; Shelke, K. F.; Shingate, B. B.; Shingare, M. S. Tetrahedron Lett. 2009, 50, 1754. (b) Niralwad, K. S.; Shingate, B. B.; Shingare, M. S. Ultrason. Sonochem. 2010, 17, 760. (c) Shinde, P. V.; Sonar, S. S.; Shingate, B. B.; Shingare, M. S. Tetrahedron Lett. 2010, 51, 1309. (d) Niralwad, K. S.; Shingate, B. B.; Shingare, M. S. Tetrahedron Lett. 2010, 51, 3616.

37. Loupy, A. Solvent-free Reactions In Modern Solvents In Organic Synthesis; Knochel, P., Ed.; Topics in Current Chemistry 1999, 206, 153.

38. De La Guardia, M.; Salvador, A. Analytica Chimica Acta 1989, $224,123$. 